\title{
POLARIZATION-CORRELATION MICROSCOPY OF HUMAN LIQUID POLYCRYSTALLINE FILMS IN INFERTILITY DIAGNOSIS
}

\author{
V.O. Ushenko ${ }^{1}$, V.P. Prysyazhnyuk ${ }^{2}$, O.V. Dubolazov ${ }^{1}$, A.O. Karachevtsev ${ }^{1}$, O.I. Olar ${ }^{2}$, \\ O.V. Olar' ${ }^{2}$, Yu.F. Marchuk ${ }^{2}$, V.O.Savich ${ }^{2}$ \\ ${ }^{1}$ Optics and Publishing Department, Chernivtsi National University, \\ 2 Kotsyubinsky Str., Chernivtsi, 58012, Ukraine \\ ${ }^{2}$ Bukovinian State Medical University, Chernivtsi, 58000, Ukraine
}

a.dubolazov@,chnu.edu.ua

\begin{abstract}
A new azimuthally stable polarimetric method for processing of microscopic images of optically anisotropic structures of different biological layers blood plasma is proposed. A new model of phase anisotropy definition of biological tissues by using superposition of Mueller matrices of linear birefringence and optical activity is proposed. The matrix element $M_{44}$ has been chosen as the main information parameter, which value is independent of rotation angle of both sample and probing beam polarization plane.
\end{abstract}

Keywords: polarimetry, blood plasma, laser image, filtering.

\section{INTRODUCTION}

Development of computational methodologies for processing of microscopic images is a new health-physical method. It is laser polarimetry of biological tissues histological sections [1 - 3]. It is based on measurement of the coordinate distributions (polarization maps) in the plane of polarization states of histological sections microscopic images of biological tissues. This method allows a new, inaccessible to the histological and mathematical methods of analysis, information on the optical anisotropy (linear and circular birefringence) multiscale structural elements of different biological objects.

At the same time, a complex analysis of the polarization maps of a tissue specimen is azimuthally dependent to the probing beam polarization plane and sample rotation angle. This makes it difficult to use this method in comparative research groups histological sections with different pathologies. Thus, further progress of laser polarimetry may be connected with the development of azimuthally stable methods of direct measurement of the parameters of linear and circular birefringence.

The solution of this task is using of Mueller-matrix cartography with so-called rotational invariants. It has been shown in the works $[4-6]$ that azimuthally stable, independent of the sample rotation angle $(\Theta)$ are the following matrix elements $M_{44}(\Theta)=$ const

It has been determined that in the case of optically thin (attenuation coefficient $\tau \leq 0.1$ ) layers the $M_{44}(\Theta)$ value is preferably defined by phase anisotropy mechanisms - linear and circular birefringences [7-21].

Applications of Digital Image Processing XXXVIII, edited by Andrew G. Tescher, Proc. of SPIE Vol. 9599, 959922 - ( ) 2015 SPIE · CCC code: 0277-786X/15/\$18

doi: $10.1117 / 12.2187448$

Proc. of SPIE Vol. $9599959922-1$ 
The separation of such mechanisms $\left(M_{44} \Rightarrow\left(\begin{array}{c}M_{44}-\text { linear } \\ M_{44}-\text { circular }\end{array}\right)\right.$ ) is possible with the help of spatial-frequency Fourier selection $[12,13]$.

Our research is aimed at designing the experimental method of azimuthally stable Fourier's polarimetry and spatial-frequency selection of parameter distributions of linear and circular birefringence of the blood plasma donors films and patients with liver cirrhosis.

\section{THE THEORY OF METHOD}

The following model concepts have been assumed [1-3] by us as the basis for an analysis of the processes of modulation of laser radiation by the polycrystalline network of biological crystals with linear and circular birefringence:

- blood plasma consists of two optical anisotropic components - globulin and albumin crystals;

- the optical anisotropic albumin network is formed by large-scale (the range of the transverse dimensions $l \sim 30 \mu m \div 100 \mu m$ ) crystals with a prevalence by linear birefringence;

- optical anisotropy of these structures is characterized by the distribution of the coordinate matrix element, where the - direction of the optical axis, - phase shift between linearly polarized orthogonal components of the light beam amplitude;

- the optical anisotropic component of the globulin is formed by spherulitic crystals $(l \sim 5 \mu m \div 10 \mu m ; L \approx l)$ with predominantly circular birefringence $M_{44}(\theta)$, where $\theta$ - polarization plane rotation angle.

\section{OPTICAL REALIZATION OF SPATIAL-FREQUENCY FOURIER'S SELECTION OF THE POLYCRYSTALLINE NETWORK}

Fig. 1 presents a diagram of laser Fourier polarimeter with spatial-frequency filtration [9].

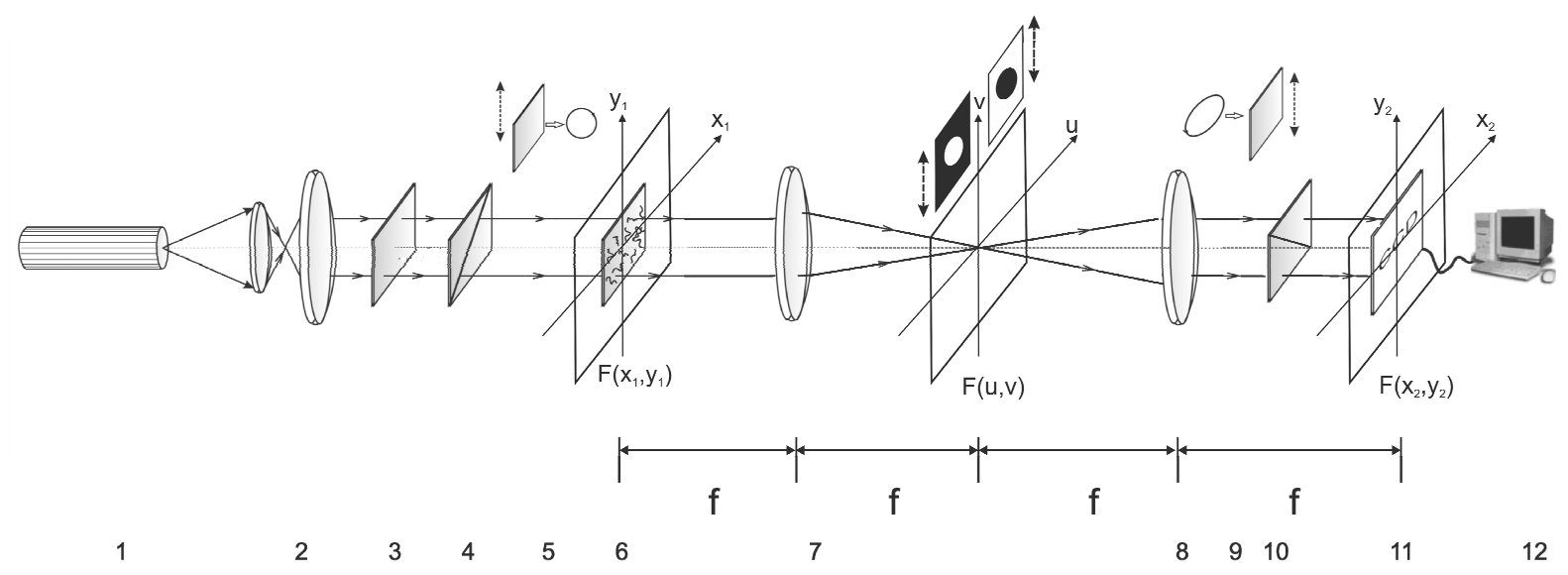

Fig. 1. Optical scheme of Fourier polarimeter, where 1 - He-Ne laser, 2 - a collimator, 3 - a stationary quarter-wave plate, 5, 9 - mechanically movable quarter-wave plates, 4, 10 - a polarizer and analyzer respectively; 6 - object of study 7,8-polarization microobjectives; 11 - CCD camera; 12 - a personal computer. $F\left(x_{1}, y_{1}\right)$ - object plane, $F\left(x_{2}, y_{2}\right)$

- image plane, $F(u, v)$ - Fourier plane with the diaphragms

Illumination of a sample under study was performed by the parallel $\left(\varnothing=10^{4}\right)$ laser beam of He-Ne $(\lambda=0.6328$ $\mu m, \mathrm{~W}=5.0 \mathrm{~mW}$ ). The polarization light source consisted of quarter-wave plates $3 ; 5$ and polarizer 4 , it formed a right circularly polarized beam. Blood plasma films were placed in the focal plane of polarization microobjective 7 (focal distance $-30 \mathrm{~mm}$, aperture -0.1 , magnification $-4 \mathrm{x}$ ). Behind the (Fourier) focal plane the vignetting diaphragm was located, its size changed within the range of 2 pix to 300 pix. Polarization microobjective 8 (focal distance $-30 \mathrm{~mm}$, aperture -0.1 , magnification $-4 \mathrm{x}$ ) was located at the focal length form the frequency plane of lens 7 and, thus, 
performed inverse Fourier transform of a filtered out polarization field of laser radiation. The coordinate distribution of intensity of such fields, polarizationally filtered by quarter-wave plate 9 and polarizer 10, was registered in the plane of CCD-camera 11 (The Imaging Source DMK 41AU02.AS, monochrome 1/2" CCD, Sony ICX205AL (progressive scan); resolution - 1280x960; light sensitive area size $-7600 \times 6200 \mu \mathrm{m}$; sensitivity -0.05 lx; dynamic range -8 bit; SNR -9 bit, deviation of photosensitive characteristics from linear no more then $15 \%$ ). It provided the range of measuring the structural elements of polycrystalline network with the resolution of 2-2000 $\mu \mathrm{m}$ [18-21].

Matrix element $M_{44}$ was calculated for each probing beam within every pixel $(m \times n)$

$$
M_{44}=\frac{I_{\circledast}^{\otimes}-I_{\oplus}^{\otimes}}{I_{\circledast}^{\otimes}+I_{\oplus}^{\otimes}}-0.5\left(\frac{I_{\circledast}^{0}-I_{\oplus}^{0}}{I_{\otimes}^{0}+I_{\oplus}^{0}}+\frac{I_{\circledast}^{90}-I_{\oplus}^{90}}{I_{\circledast}^{90}+I_{\oplus}^{90}}\right) .
$$

Here $I_{\otimes}^{\otimes ; 0 ; 90} ; \quad I_{\oplus}^{\otimes ; 0 ; 90}$ - are the intensities of spatial-frequency filtered image $(\otimes ; \quad \oplus)$ for each polarization states of probing beam $\left(\otimes ; \quad 0^{0} ; \quad 90^{0}\right)$.

To quantify the coordinate distributions we used statistical analysis [7-10]. We calculated a set of statistical moments of the 1-st - 4-th orders $Z_{j=1 ; 2 ; 3 ; 4}$ using the following algorithms

$$
Z_{1}=\frac{1}{N} \sum_{i=1}^{N}\left|(q)_{i}\right|, Z_{2}=\sqrt{\frac{1}{N} \sum_{i=1}^{N}(q)_{i}^{2}}, Z_{3}=\frac{1}{\left(Z_{2}\right)^{3}} \frac{1}{N} \sum_{i=1}^{N}(q)_{i}^{3}, Z_{4}=\frac{1}{\left(Z_{2}\right)^{4}} \frac{1}{N} \sum_{i=1}^{N}(q)_{i}^{4} .
$$

The obtained data indicate the distribution histogram of random values optically anisotropic structures in the plane of the histological section.

\section{EXPERIMENTAL RESULTS AND DISCUSSION}

As objects of investigation, we chose two groups of optically-thin (attenuation coefficient $\tau \approx 0,087 \div 0,098$ ) single scattered blood plasma films patients with mycoplasmosis (36 samples - group 1) and patients with chlamydia (36 samples - group 2).

Fig. 2 shows the classic microscopic images of samples of both groups.

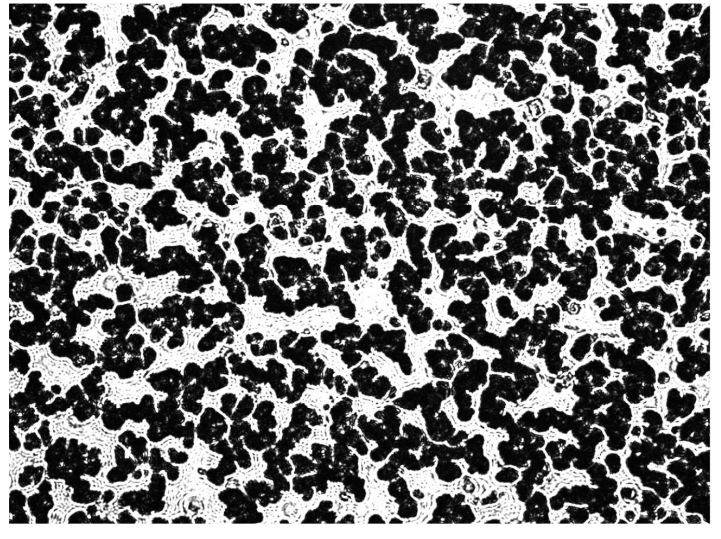

a)

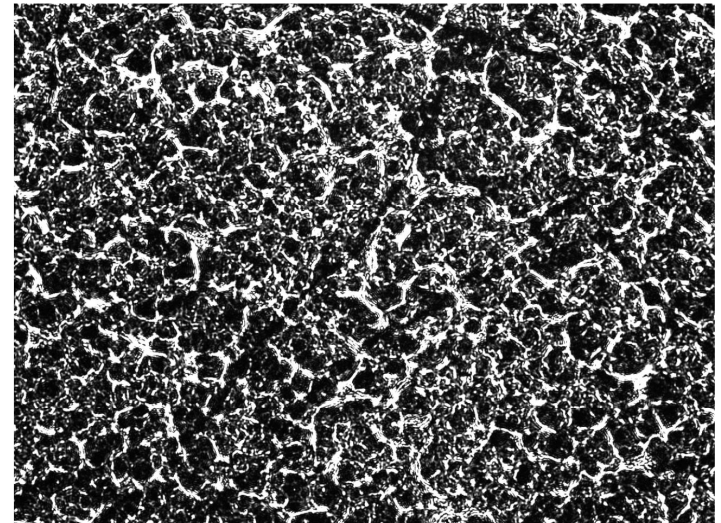

b)

Fig. 2. Microscopic images of the blood plasma films: group 1 (a) and group 2 (b)

As can be seen, coordinate large-scale structure of such classic microscopic images is similar. This fact makes it difficult to histological differentiation of blood plasma films. 


\section{SPATIAL-FREQUENCY FOURIER POLARIMETRY OF LINEAR BIREFRINGENCE OF THE ENDOMETRIUM}

For the purpose of choosing optimal conditions of spatial-frequency filtration the following range $\Delta r=2 p i x \div 50$ pix of possible sizes of the vignetting diaphragm.

The criterion for diaphragm size choosing is simultaneous change of the set of statistic moments $Z_{j=1 ; 2 ; 3 ; 4}$ (4) of $M_{44}$. In our case the optimal size was $\Delta r=30$ pix.

Such geometric size was chosen for comparative investigations of optical anisotropy of the fibrilar networks of the blood plasma films, which characterize by coordinate distributions of rotational invariant $M_{44}(\delta)$ - Fig.3.

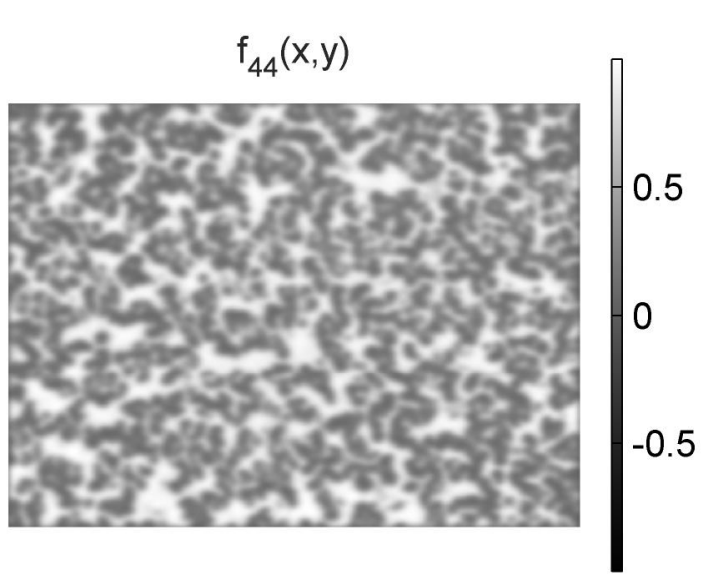

1)

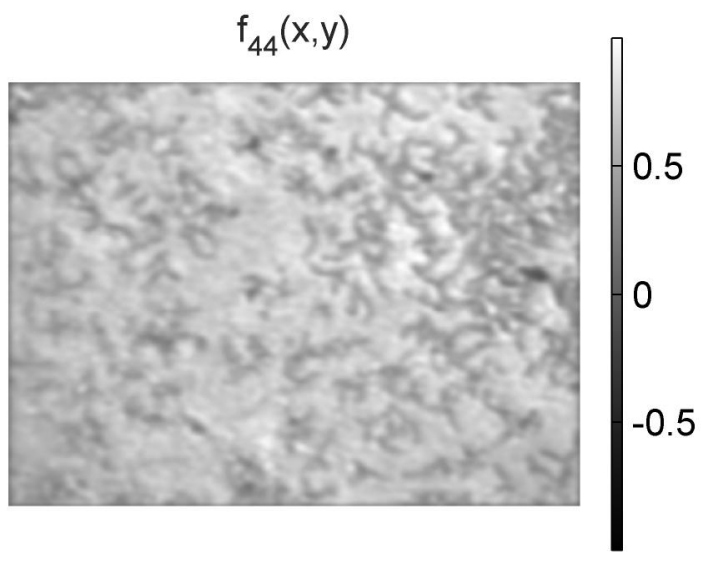

3)

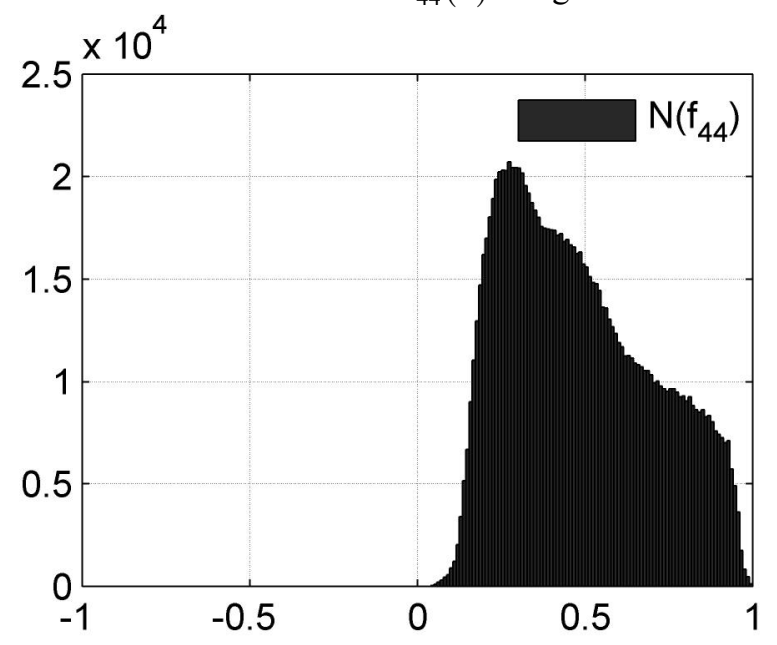

2)

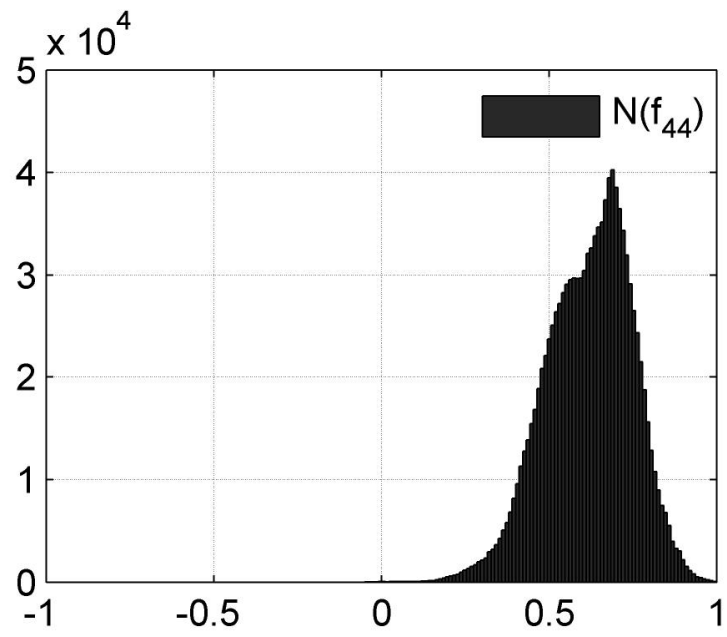

4)

Fig. 3. "Low-frequency" coordinate maps $M_{44}(\delta)(1),(3)$ histograms (2),(4) of the linear birefringence distribution of blood plasma films of group $1((1),(2))$ and group $2((3),(4))$. 
A comparative analysis of aggregate parameters, that characterize matrix maps $M_{44}(\delta)$ of linear birefringence of large-scale optically anisotropic networks of the plasma samples, revealed some discrepancies between them. Namely, the main extrema of histograms of the distributions of random matrix maps $M_{44}(\delta)$ values for histological sections of both types are localized in different areas. Thus, the most probable is value $M_{44}(\delta) \sim 0.3$ is for a group 1 (fig. 3,(2)); for a sample $2 M_{44}(\delta) \sim 0.6$ (fig. 3,(4)). The revealed peculiarity, in our opinion, is connected with a more developed structure of the blood plasma films of ill patient. Quantitatively differences between matrix maps $M_{44}(\delta)$ of the protein matrices of the blood plasma films of both types illustrate average values and standard deviations of the set of statistic $Z_{i=1 ; 2 ; 3 ; 4}$ parameters, illustrated in the table 1 . Also, let us introduce the specificity parameter $S p=a(a+b)^{-1}(a, b-$ the number of the correct and incorrect diagnoses within group) in differentiation of normal - liver cirrhosis.

Table 1. Parameters of the statistic structure of matrix maps $M_{44}(\delta)$ of linear birefringence of blood plasma films

\begin{tabular}{|c|c|c|c|}
\hline \multirow{2}{*}{ Parameters } & \multicolumn{2}{|c|}{$M_{44}(\delta)$} & \multirow{2}{*}{$S p$} \\
\cline { 2 - 3 } & Group 1 & Group 2 & \\
\hline$Z_{1}$ & $0,23 \pm 0,059$ & $0,52 \pm 0,11$ & $78 \%$ \\
\hline$Z_{2}$ & $0,12 \pm 0,022$ & $0,15 \pm 0,031$ & $63 \%$ \\
\hline$Z_{3}$ & $1,26 \pm 0,37$ & $0,78 \pm 0,14$ & $83 \%$ \\
\hline$Z_{4}$ & $1,59 \pm 0,28$ & $1,13 \pm 0,19$ & $79 \%$ \\
\hline
\end{tabular}

A comparative analysis of the data of laser-frequency Fourier's - polarimetry of linear birefringence of blood plasma films of both groups of patients revealed sensibility to a differentiation of such states of all statistic moments of the $1^{\text {st }}$ through the $4^{\text {th }}$ orders with specificity level $S e \sim 78 \%-83 \%$ (printed in grey color in table 1 ).

\section{CONCLUSIONS}

A set of criteria for a polarization-phase differentiation of blood plasma films has been revealed and substantiated: - statistical moments of the $1^{\text {st }}-4^{\text {th }}$ order which characterize a distribution of azimuthally stable matrix element $M_{44}(\delta)$, stipulated by linear birefringence of the blood plasma films;

- statistical moments of the $1^{\text {st }}-4^{\text {th }}$ orders which characterize a distribution of azimuthally stable matrix element $M_{44}(\theta)$, stipulated by circular birefringence of the blood plasma films.

\section{Acknowledgement}

This work was supported by the grants from the Ukraine Foundation for Basic Research.

\section{REFERENCES}

[1]. T.T. Tower, R.T.Tranquillo, "Alignment Maps of Tissues: I. Microscopic Elliptical Polarimetry," Biophys. J. 81, 2954-2963 (2001).

[2]. Shribak. M., Oldenbourg. R., "Techniques for Fast and Sensitive Measurements of Two-Dimensional Birefringence Distributions," Appl. Opt. 42, p.p. 3009-3017 (2003).

[3]. Smith, M.H, "Interpreting Mueller matrix images of tissues," Proc. SPIE 4257, p.p.82-89 (2001).

[4]. Ushenko, A.G., Misevich, I.Z., Istratiy, V., Bachyns'ka, I., Peresunko, A.P., Numan, O.K., Moiysuk, T.G., "Evolution of statistic moments of 2D- distributions of biological liquid crystal net mueller matrix elements in the process of their birefringent structure changes ," Advances in Optical Technologies, art. no. 423145 (2010).

[5]. Angelsky, O.V., Bekshaev, A.Ya., Maksimyak, P.P., Maksimyak, A.P., Hanson, S.G., Zenkova, C.Yu., "Selfdiffraction of continuous laser radiation in a disperse medium with absorbing particles," Optics Express 21(7), p.p. $8922-8938$ (2013).

[6]. Angelsky, O. V., Ushenko, A. G., Burcovets, D. N., Ushenko, Yu. A., "Polarization visualization and selection of biotissue image two-layer scattering medium," J. Biomed. Opt. 10(1), P. 014010 (2005). 
[7]. Ushenko, A., Yermolenko, S., Prydij, A., Guminetsky, S., Gruia, I., Toma, O., Vladychenko, K. "Statistical and fractal approaches in laser polarimetry diagnostics of the cancer prostate tissues," Proceedings of SPIE - The International Society for Optical Engineering, 7 008, art. no. 7008 2C (2008).

[8]. Olar, E.I., Ushenko, A.G., Ushenko, Yu.A., "Correlation microstructure of the Jones matrices for multifractal networks f biotissues," Laser Physics, 14 (7), pp. 1012-1018 (2004).

[9]. Ushenko, A.G., Burkovets, D.N., Ushenko, Yu.A., "Polarization - Phase Mapping and Reconstruction of Biological Tissue Architectonics during Diagnosis of Pathological Lesions", Optics and Spectroscopy, 93 (3), pp. 449-456 (2002).

[10]. Angelsky, O.V., Gorsky, M.P., Hanson, S.G., Lukin, V.P., Mokhun, I.I., Polyanskii, P.V., Ryabiy, P.A., "Optical correlation algorithm for reconstructing phase skeleton of complex optical fields for solving the phase problem," Optics Express, Vol. 22 (5), pp.6186-6193 (2014).

[11]. Angelsky, O.V., Zenkova, C.Y., Gorsky, M.P., Gorodyns'ka, N.V., "Feasibility of estimating the degree of coherence of waves at the near field," Applied optics 48 (15), 2784-2788 (2009).

[12]. Ushenko, A.G., "Polarization Contrast Enhancement of Images of Biological Tissues under the Conditions of Multiple Scattering," Optics and Spectroscopy, 91 (6), pp. 937 -940 (2001).

[13]. Ushenko, A.G., "Laser Probing of Biological Tissues and the Polarization Selection of Their Images," Optics and Spectroscopy, 91 (6), pp. 932-936 (2001).

[14]. Angel'skiǐ, O.V., Ushenko, A.G., Ermolenko, S.B., Burkovets,D.N., Ushenko, Yu.A., Pishak, O.V., "PolarizationBased Visualization of Multifractal Structures for the Diagnostics of Pathological Changes in Biological Tissues," Optics and Spectroscopy (English translation of Optika i Spektroskopiya) 89 (5), p.p. 799-804 (2000).

[15]. Ushenko, Y.A., Dubolazov, A.V., Angelskya, A.P., Sidor, M.I., Bodnar, G.B., Koval, G., Zabolotna, N.I., "Laser polarization fluorescence of the networks of optically anisotropic biological crystals," Optical Fibers and Their Applications, Proc. of SPIE Vol. 8698 869809-1 (2012).

[16]. Angelsky, O.V., Ushenko, A.G., Ushenko Y.A., "Polarization reconstruction of orientation structure of biological tissues birefringent architectonic nets by using their Mueller-matrix speckle-images," Journal of Holography and Speckle 2 (2), 72-79 (2005).

[17]. Angelsky, O.V., Ushenko, A.G., Ushenko Y.A., Pishak, V.P., Peresunko A.P., [Statistical, correlation, and topological approaches in diagnostics of the structure and physiological state of birefringent biological tissues], Handbook of Photonics for Biomedical Science, 21-67 (2010).

[18]. Ushenko, A.G., Burkovets, D.N., Ushenko Y.A., "Polarization-phase mapping and reconstruction of biological tissue architectonics during diagnosis of pathological lesions,"Optics and Spectroscopy 93 (3), 449-456 (2002).

[19]. Angelsky, O.V., Maksimyak, P.P., Perun, T.O., "Dimensionality in optical fields and signals," Applied Optics, v.32 (30), pp.6066-6071 (1993).

[20]. Ushenko, Yu.A., Sidor, M.I., Pashkovskaia, N., Koval, G.D., Marchuk Yu.F., Andreichuk D., "Laser polarizationvariable autofluorescence of the network of optically anisotropic biological tissues: diagnostics and differentiation of early stages of cancer of cervix uteri," Journal of Innovation Optical Health Sciences. Vol. 7,№3 1450024 (2014).

[21]. Angelsky, O.V., Bekshaev, A.Ya., Maksimyak, P.P.,; Maksimyak, A.P., Hanson, S.G.,; Zenkova, C. Yu., "Selfaction of continuous laser radiation and Pearcey diffraction in a water suspension with light-absorbing particles," Optics Express, Vol. 22 (3), pp. 2267-2277(2014). 